\title{
Feasibility of laparoendoscopic single-site surgery in supracervical hysterectomy: technique and retrospective case-control comparison
}

\author{
Per Istre • Lars Franch Andersen • \\ Henrik Halvor Springborg
}

Received: 13 May 2014 / Accepted: 19 January 2015 /Published online: 3 February 2015

(C) Springer-Verlag Berlin Heidelberg 2015

\begin{abstract}
One recent innovation in the field of gynecology is laparoendoscopic single-site surgery (LESS). It is associated with reduced postoperative pain and better cosmetic outcome. The present paper aims to explain techniques and instrumentations associated with single-port hysterectomies. A retrospective case-control study is included, comparing LESS with conventional four-port hysterectomies to highlight the feasibility of LESS. This study involved literature search and personal experience regarding single-port hysterectomy and a retrospective case-control study of 34 patients who underwent supracervical hysterectomy between April 2011 and November 2012. Operating time, blood loss, and length of hospital stay were similar in the two groups. Patient's evaluation of the cosmetic result was in favor of LESS, however not significant. LESS represents a new frontier in minimally invasive surgery. New instrumentation and recommendations have been developed which are expected to make the technique more feasible. This study demonstrates that LESS supracervical hysterectomy has comparable operative outcomes to conventional laparoscopic hysterectomy and is a feasible approach for supracervical hysterectomy. Based on the literature and our experience, a feasible step by step technique for surgeons who are interested in performing supracervical LESS
\end{abstract}

\footnotetext{
P. Istre $(\bowtie)$

Department of Gynecology/Obstetrics, Hvidovre University

Hospital, Kettegård Allé 30, 2650 Hvidovre, Denmark

e-mail: pistre@gmail.com

L. F. Andersen

Department of Gynecology/Obstetrics, Nordsjællands Hospital

Hillerød, Dyrehavevej 29, 3400 Hillerød, Denmark

e-mail: lars.franch.andersen@dadlnet.dk

H. H. Springborg

Department of Gynecology, Aleris-Hamlet Private Hospital,

Gyngemose Parkvej 66, 2860 Søborg, Denmark

e-mail: halvor-springborg@tdcadsl.dk
}

hysterectomy is described. Comparative data and prospective trials are required in order to determine the clinical utility and impact of LESS in treatment of gynecological conditions in the future.

Keywords LESS · Single-port laparoscopy \pm hysterectomy · Conventional laparoscopy \pm hysterectomy $\cdot$ Single-port laparoscopy versus conventional laparoscopic hysterectomy

\section{Introduction}

Hysterectomy is among the most commonly performed gynecologic surgeries. The method of hysterectomy has evolved during the recent years from laparotomy to laparoscopic procedures which continuously are being improved [1]. The advantages of a minimally invasive laparoscopic approach compared to traditional laparotomy are reduced postoperative pain, reduced perioperative bleeding, reduced risk of infection, faster recovery, better cosmetic results, and shorter hospital stay $[2,3]$. Current efforts aim to reduce perioperative morbidity associated with laparoscopic surgery. One of the more recent innovations in the field of minimally invasive surgery is laparoendoscopic single-site surgery (LESS). This technique allows the surgeon to perform the abdominal intervention through a single incision in the navel, as compared to the traditional multiport laparoscopic surgical technique, which requires three or more abdominal incisions [4]. Each working port has an inherent risk of bleeding, infection, organ damage, hernia formation, and decreased cosmetics; the ideal goal of laparoscopy is to reduce port number and port size [5].

LESS technique has been implemented in urology, gastrointestinal surgery, and gynecology in an attempt to reduce abdominal wall trauma [6]. Evidence suggests that singleport laparoscopic hysterectomy (LESSH) is a feasible and safe 
approach $[1,4,5,7,8]$. However, it remains to be seen whether there are significant benefits of LESSH compared to conventional laparoscopic hysterectomy (CLH).

The technical challenges of LESSH include limited triangulation and compromised retraction due to the limitation of the instruments through a single axis. Due to instrumental angulation, surgeons may encounter difficulty with suturing, requiring a greater level of surgical expertise. One significant finding has been the extended operation time performing LESSH $[4,9]$. However, studies show that with increasing operator experience, operative time can be reduced $[1,4]$.

In gynecology, supracervical hysterectomy has been attractive for patients with fibroids or abnormal bleeding and the absence of a specific indication for removal of the cervix, citing lower complication rates (less bleeding, fewer infections/abscesses and vaginal cuff hematomas, trauma to urinary tract), shorter operative times, less postoperative pain, faster recovery, and a less complicated procedure. Arguments against the supracervical procedure are the potential for bleeding from residual endometrium in the cervical stump, absence of using the vagina as a port for specimen removal, the need for a continued cervical screening program, and the potential risk of future cervical pathology [10-14].

Subjective and objective outcomes such as less postoperative pain and improved cosmetic results have been associated with single-port surgery. It has been suggested that these findings can be partly explained by avoidance of the secondary ports penetrating abdominal muscles [15-17]. The present paper aims to explain techniques and instrumentations associated with single-port hysterectomies. A retrospective casecontrol study is included, comparing LESS with conventional four-port hysterectomies to highlight the feasibility of LESS (Fig. 1).

\section{Material and methods}

Methods

Patients were recruited from the primary health care sector by specialists in gynecology and were referred to the Minimal Invasive Gynecological Surgery (MIGS) unit at AlerisHamlet Hospital in Copenhagen, Denmark. All patients underwent pelvic examination and transvaginal ultrasound in the outpatient clinic. Patients were then scheduled for surgery within 3 weeks. Inclusion criteria for the LESSH procedure were $\mathrm{BMI}<35$, ultrasound-estimated uterine weight $<300 \mathrm{~g}$, no suspicion of malignancy and periumbilical adhesions, and American Society of Anesthesia (ASA) I-II. If a patient met the inclusion criteria for the LESSH procedure, she was offered a choice between the two types of laparoscopic procedures: LESSH or CLH. The patients were informed that CLH was the standard procedure at the department and that the LESSH technique was a new but feasible technique, where studies indicated that the safety was comparable to CLH. The patients were further informed that the LESSH procedure
Fig. 1 a Conventional laparoscopy, using four ports. b Camera, cutting forceps, and grasper in three different ports during single-port laparoscopy. c Cosmetic result immediately after a single-port laparoscopy

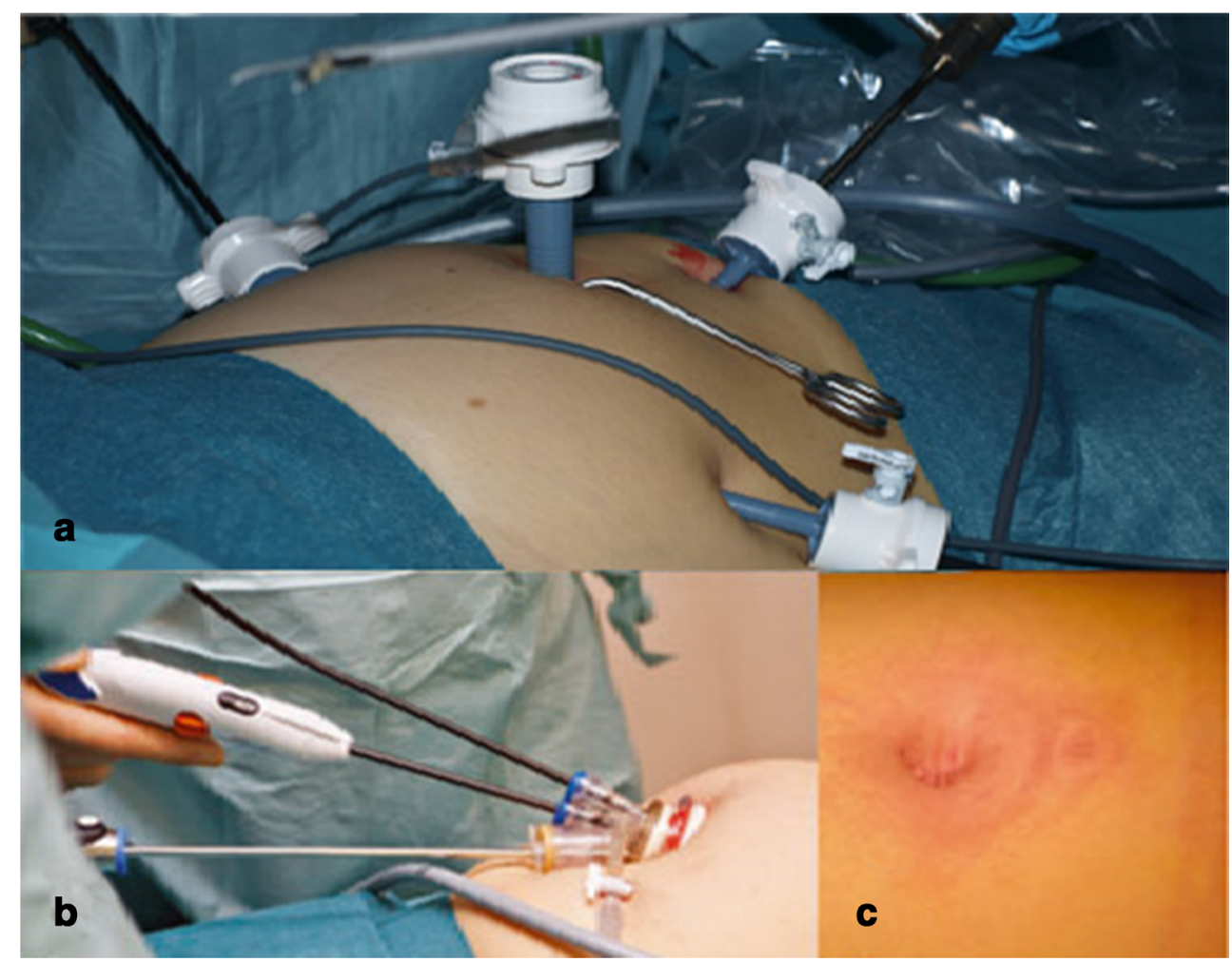


might have cosmetic advances since the scar is partly hidden in the umbilicus.

To make the two procedures LESSH and CLH as comparable as possible for this study, only supracervical operations were included.

\section{Study design}

A case-control study including 34 performed supracervical hysterectomies between April 4, 2011, and November 16, 2012, was conducted. Based on the surgeon's explanation and preoperative discussion, the patients freely chose their preferred operation. Each corresponding CLH which met given LESSH inclusion criteria was picked after each selected LESSH, in order to make the two groups comparable. All operations were performed by the department's two experienced surgeons.

The primary endpoint of this retrospective study was to compare operative details, operation time, blood loss, and length of hospital stay between the two groups, as well as the technical difficulties with the procedures. Secondary endpoint was to evaluate patient satisfaction using a questionnaire sent out to the patients. The questionnaire focused on the time interval before returning to everyday activities and work and also the patient's evaluation of the performed operative procedure and cosmetic outcome.

\section{Data collection}

Clinical characteristics age, BMI, ASA, and indication for surgery and surgical characteristics operation time, blood loss, uterine weight, and length of hospital stay were recorded for each patient. Among the 34 patients, 27 completed the questionnaire in which they assessed the cosmetic result, overall satisfaction with the performed surgery, and whether they would recommend it to others. The excluded seven patients had either moved from the country, had an invalid telephone number, or were unreachable after repeated attempts to contact them during 1 month's time.

\section{Statistics}

Data were collected and processed using IBM SPSS v20 ${ }^{\circledR}$. Descriptive analytics included percentage, median, and range. Differences between the two groups were analyzed using the Mann-Whitney $U$ test for nonparametric distribution. A $p$ value $<0.05$ was considered to indicate statistical significance.

\section{Results}

A total of 34 supracervical laparoscopic hysterectomies were included in this study, with 17 patients in each group: LESSH and CLH. Indication for hysterectomy included uterine fibroids, $n=20(58 \%)$; lower abdominal pain from hematometra after endometrial resection, $n=4(11 \%)$; dysmenorrhea, $n=4$ (11\%); menorrhagia, $n=3$ ( $8 \%$ ); and metrorrhagia, $n=2(6 \%)$. Age, BMI, and ASA-value showed no statistical differences between the two groups. No LESSH operation was converted to CLH or laparotomy, and no CHL procedure was converted to laparotomy (Table 1).

Surgical outcomes are shown in Table 2: median operation time of $70 \mathrm{~min}$, blood loss of $30 \mathrm{~mL}$, and uterine weight of $140 \mathrm{~g}$ in the LESSH group compared to $70 \mathrm{~min}, 30 \mathrm{~mL}$, and $130 \mathrm{~g}$ in the CLH group, respectively. Six patients from the LESSH group were discharged from hospital on the day of operation, compared with two patients from the CLH group. The majority of patients, 10 patients in the LESSH group and 15 in the CLH group, were discharged the next day. One patient in the LESSH group stayed more than 1 day because of postoperative nausea. There were no significant differences between the two groups in any of the parameters: duration of surgery, blood loss, and uterus weight. Reviewing LESSH, the median duration of surgery, blood loss, and uterus weight showed no significant difference between the first eight patients, compared to the next nine patients (Table 3).

Median time before returning to work was 14 days for patients in LESSH group and 10 for the CLH group. The median scores for cosmetic result, overall assessment, and if they were to recommend surgery for others were 10,10 , and 10 , respectively, in the LESSH group against 9, 9, and 10 in the CLH group. No significant difference was found between the two groups for any of these parameters (Table 4).

Table 1 Demographic and clinical characteristics of patients who underwent either single-port laparoscopic hysterectomy (LESSH) or conventional laparoscopic hysterectomy (CLH), both supracervical hysterectomies for various benign gynecological diseases

\begin{tabular}{|c|c|c|c|}
\hline Characteristics & $\begin{array}{l}\text { LESSH } \\
(N=17)\end{array}$ & $\begin{array}{l}\text { CLH } \\
(N=17)\end{array}$ & $\begin{array}{l}\text { Total } \\
(N=34)\end{array}$ \\
\hline Age, years & $45(39-53)$ & $45(39-72)$ & $45(39-72)$ \\
\hline Body mass index, $\mathrm{kg} / \mathrm{m}^{2}$ & $24(19-32)$ & $24(19-34)$ & $24(19-34)$ \\
\hline \multicolumn{4}{|l|}{ ASA grading } \\
\hline 1 & $14(82)$ & $11(71)$ & $25(76)$ \\
\hline 2 & $3(18)$ & $6(29)$ & $9(24)$ \\
\hline \multicolumn{4}{|l|}{ Indication of surgery } \\
\hline Lower abdominal pain & $0(0)$ & $1(5)$ & $1(3)$ \\
\hline Dysmenorrhea & $1(5)$ & $3(17)$ & $4(11)$ \\
\hline Uterine fibroid & $9(52)$ & $11(64)$ & $20(58)$ \\
\hline Menorrhagia & $3(17)$ & $0(0)$ & $3(8)$ \\
\hline Metrorrhagia & $1(5)$ & $1(5)$ & $2(6)$ \\
\hline Hematometra & $3(17)$ & $1(11)$ & $4(11)$ \\
\hline
\end{tabular}

Data presented as median (range) or $n(\%)$ patients

ASA American Society of Anesthesia 
Table 2 Surgical outcomes for patients who underwent either singleport laparoscopic hysterectomy (LESSH) or conventional laparoscopic hysterectomy (CLH), as supracervical hysterectomies for various benign gynecological diseases

\begin{tabular}{lrrr}
\hline Parameter & LESSH $(N=17)$ & CLH $(N=17)$ & $p$ value $^{\mathrm{a}}$ \\
\hline Duration of surgery, min & $70(45-100)$ & $70(40-105)$ & 0.375 \\
Blood loss, mL & $30(10-200)$ & $30(5-150)$ & 0.946 \\
Uterus weight, g & $140(50-290)$ & $130(63-300)$ & 0.760 \\
Postoperative hospital stay, days & & \\
Same day & 6 & 2 & \\
1 day & 10 & 15 & \\
More than 1 day & 1 & 0 & \\
\hline
\end{tabular}

Data is presented as median (range) or numbers

${ }^{\text {a }}$ Mann-Whitney $U$ test.

\section{Discussion}

Recent advances in laparoscopic equipment and improvements in surgical skills have further enhanced the advantages of laparoscopic surgery. Many surgeons have attempted to reduce abdominal wall trauma by minimizing the number of ports and the size of instruments. A limited number of studies suggest that using a single-port entrance is associated with fewer days of immobilization and higher rates of patient satisfaction $[1,18]$. This study may indicate likewise, as many patients who underwent the LESS procedure were discharged from hospital the same day as the operation.

LESSH patients' median score in this study on the questions concerning patient satisfaction, including cosmetic outcome, was 10, whereas CLH patients' median score was 9. This difference was not statistically significant; however, the score suggests that LESS might be advantageous cosmetically. A cross-sectional study done by Eom et al. [3] and a more recent randomized study by Song et al. [17] showed better cosmetic result and overall satisfactory rate by patients undertaking the LESS procedure compared to the conventional procedure.

The LESSH procedure has the disadvantage of restricted movements of surgical instruments due to the proximity of the instruments [2]. One of the indicators found to be significant in the majority of earlier studies was prolonged operation

Table 3 Surgical outcomes of the first eight patients who underwent single-port laparoscopic hysterectomy (LESSH), compared with the next nine patients who underwent the same procedure

\begin{tabular}{llll}
\hline Parameter & First 8 patients & Next 9 patients & $p$ value $^{\mathrm{a}}$ \\
\hline Duration of surgery, min & $67.5(57-95)$ & $70(45-100)$ & 0.673 \\
Blood loss, mL & $30(15-200)$ & $30(10-100)$ & 0.673 \\
Uterus weight, g & $140(70-251)$ & $135(50-290)$ & 0.743 \\
\hline
\end{tabular}

Data is presented as median (range)

${ }^{\text {a }}$ Mann-Whitney $U$ test
Table 4 Questionnaire outcome from the patients of the two different groups: LESSH and CLH

\begin{tabular}{llll}
\hline Parameter & $\begin{array}{l}\text { LESSH } \\
(N=12)\end{array}$ & $\begin{array}{l}\text { CLH } \\
(N=15)\end{array}$ & $p$ value $^{\mathrm{a}}$ \\
\hline Everyday activities, days & $6(2-14)$ & $4(1-14)$ & 0.277 \\
Resuming to work, days & $14(3-42)$ & $10(2-21)$ & 0.516 \\
Overall assessment, score (1-10) & $10(8-10)$ & $9(2-10)$ & 0.399 \\
Cosmetic outcome, score (1-10) & $10(9-10)$ & $9(3-10)$ & 0.167 \\
Recommend surgery, score (1-10) & $10(9-10)$ & $10(8-10)$ & 0.427 \\
\hline
\end{tabular}

In the scoring procedure, 1 indicates unsatisfactory, while 10 satisfactory. Data is presented as median (range)

${ }^{\text {a }}$ Mann-Whitney $U$ test

time. Lack of surgical experience and the need for new instruments with more flexibility have been suggested as the causes for the extended operating times with the single-port technique $[1,4,9]$. In this study, no difference in operation time was found, even when comparing the first eight patients with the next nine patients (Table 3), indicating that the learning curve was steep.

The steep learning curve may suggest the importance of following the technical recommendations from the literature. Additionally, the surgeons had experience with less complicated adnexal single-port operations on women as well as previous training in pig labs before performing hysterectomy. Lab training before performing LESSH operations is highly recommended, and simple adnexal operations are most feasible when LESSH is introduced.

The rate of complications in this study is, like in larger randomized controlled studies, low in laparoscopic hysterectomy $[2,3]$. Even though the incision in the umbilicus with LESS in several procedures is larger than in traditional multiport laparoscopy, recent literature indicates that the risks of herniation are comparable [19]. In supracervical hysterectomy involving morcellator use as in this study, the length of the incision in the umbilical fascia is comparable to traditional multiport laparoscopic hysterectomy.

Recently, there have been concerns and discussions about possible remnants of the rare but serious sarcoma fibroid tissue left behind in the abdominal cavity after power morcellation (FDA Issues Safety Communication on Laparoscopic Uterine Power Morcellation in Hysterectomy and Myomectomy 2014). The LESSH procedure may contribute to a reduction of the risk of leaving morcellated tissue behind in the abdominal cavity, as this technique facilitates morcellation of uterus inside a plastic bag in combination with the LESS triport.

The design of this retrospective case-control study implies risks of bias. Patients who met the inclusion criteria for LESSH were informed about the procedure. Patients choosing the LESSH procedure were probably more motivated to receive the most minimal surgery possible, while patients 
choosing the standard procedure $(\mathrm{CLH})$ probably were less concerned about the cosmetic outcome and preferred to undergo a procedure already well established.

To confirm the feasibility and safety of LESSH, including the risk of more rare complications and the safety of general implementation of the procedure, will require large randomized controlled studies.

\section{Conclusion}

LESSH represents a new frontier in minimally invasive surgery. New instrumentation and recommendations have been developed, which are expected to make the technique more feasible. This study demonstrates that LESSH has comparable operative outcomes to conventional laparoscopic hysterectomy and probably improved cosmetics. It is a feasible approach for supracervical hysterectomy when following recommendations from experienced surgeons in the field of LESS. Comparative data and prospective trials are required in order to determine the clinical utility and impact of LESS in treatment of gynecological conditions in the future.

\section{Equipment and technical recommendations for performing LESS supracervical hysterectomy}

The following recommendations are based on a combination of recommendations from leading surgeons in the field of LESS in the literature and lectures on conferences [20].

\section{Recommended equipment}

1. Access device of good quality like TriPort $15^{\mathrm{TM}}$ (Olympus $^{\circledR}$, Hamburg, Germany) or GelPoint ${ }^{\mathrm{TM}}$ (Applied Medical, Rancho Santa Margarita, CA) allowing an instrument of minimum of $12 \mathrm{~mm}$ for morcellation.

2. Five $\mathrm{mm} 30^{\circ}$ rotable or flex tip optics, preferably with light cable in line with the shaft of the telescope. If not available, a $90^{\circ}$ adaptor for the light cable can be used to minimize interference with the light cord.

3. Curved and/or straight grasper and straight active instrument (where the angle of the working instrument can be rotated). In order to reduce the number of instrument exchanges, a combined grasping, coagulation, and cutting instrument is recommended.

4. Uterine manipulator of good quality with a ring to delineate the vaginal fornix for better presentation of the uterine vessels.

5. For supracervical hysterectomy, no suturing is required; however, a monopolar/bipolar hook or loop (Lina loop, Lina medical ${ }^{\circledR}$, Copenhagen, Denmark) facilitated amputation of the uterus at the cervical-isthmic level.
6. A $12-15-\mathrm{mm}$ morcellator, preferably mechanical, to avoid formation of smoke during morcellation.

\section{Recommended procedures and techniques}

It is recommended to perform several simple adnexal LESS procedures before performing hysterectomy. Training in "Pig Lab" is an advantage, and performing the first LESS hysterectomies with a trained LESS surgeon is highly recommended.

1. Access technique (Hasson) - 1.5-2-cm longitudinal transumbilical skin incision and opening of the subcutaneous fat. With two Kocher clamps, the fascia is elevated and an incision is made. A blunt retractor is inserted through the peritoneum into the peritoneal cavity. The access device is placed in accordance with the manufacturer's instructions for use.

2. General procedure - after insufflation, the optic is placed in the most cephalic port with the tip close to the abdominal wall looking down and the camera head close to the chest. Then, the straight or curved grasper is inserted. Consider whether the active instrument has to pass over or under the grasper before it is introduced. It is important to maintain the laparoscope and surgical instruments in different horizontal planes in order to avoid instrument clashing. To reduce the problem of instrument clashing on the outside, the instrument handles can be kept horizontal and parallel to the floor "gangsta". Multifunctional instruments, with grasp, coagulation, and cut, reduce the number of instrument movements and exchanges and are especially helpful in LESS surgery.

3. Hysterectomy step by step (the primary surgeon on the left side of the patient and will begin the hysterectomy on the left).

a. After the camera is inserted as mentioned above, the uterus is positioned upward and toward the right by the uterine manipulator.

b. The assistant instrument (curved or straight grasper) is then inserted through the left canula and can be used to augment the positioning of the uterus to present the left utero-ovarian and broad ligaments.

c. The electrosurgical instrument is then inserted through the right canula and the utero-ovarian ligament and broad ligament including the round ligament can be sealed and transected. (When exposure is better when lateral tension on the left ovary is created, the grasper is inserted through the right canula and the active instrument through the left canula.)

d. The broad ligament is opened and the bladder peritoneum is opened from the left to the midline, and 
the peritoneum on the left side is pushed downwards. During this procedure, the uterus is positioned to the back with the uterine manipulator to expose the bladder peritoneum.

e. The left uterine vessels are sealed on the edge of the ring of the uterine manipulator in the vagina. During this procedure, the uterus is positioned upward and against the right by grasper and manipulator in order to reduce the risk of injury to the ureter.

f. The right side of the hysterectomy is performed by positioning the uterus toward the left. The grasper is positioned through the right canula and the operating instrument through the left canula. The same procedure is performed (c to e) on the right side, and the bladder is pushed down and the uterine vessels in both sides is sealed and transected.

g. The sealed uterine vessels are cut bilaterally and hemostasis ensured.

h. The loop is placed around the cervix, and it is visually secured that no bowel is close to the cutting edge. After removing the uterine manipulator, the cervix is pulled forward and away from the bowel using the loop and corpus uteri is amputated. The amputation can alternatively be performed with an electrical hook.

i. The endocervix is coagulated to reduce the risk of persisting endometrial tissue.

j. The uterus is morcellated by a $12-$ or $15-\mathrm{mm}$ morcellator through the $15-\mathrm{mm}$ port (or the GelPoint). During morcellation, it is of utmost importance that the morcellator is kept away from the bowel and close to the abdominal wall. The tip of the morcellator must at all time during morcellation be visible. A laparoscope with flexible tip is an advantage during this procedure.

$\mathrm{k}$. If problems occur, including during morcellation, an additional port should always be considered.

1. The umbilical fascia is closed with delayed absorbable suture and the skin as a separate layer with consideration of perfect adaption of skin edges to avoid secretion and achieve perfect cosmetic appearance.

On the basis of the quite substantial and promising results in the literature and by following these recommendations, we find it feasible for experienced surgeons to initiate studies and, in protocol, perform LESS supracervical hysterectomies.

Conflict of interest All of the authors contributed to the design and writing of the manuscript. Per Istre, Lars Franch Andersen, and Henrik Halvor Springborg have no conflict of interest. There are no financial disclosures. On behalf of all authors, the corresponding author states that there is no conflict of interest. Informed consent was obtained from all patients for being included in the study.

\section{References}

1. Li M, Han Y, Feng YC (2012) Single-port laparoscopic hysterectomy versus conventional laparoscopic hysterectomy: a prospective randomized trial. J Int Med Res 40(2):701-708

2. Jung YW et al (2011) A randomized prospective study of single-port and four-port approaches for hysterectomy in terms of postoperative pain. Surg Endosc 25(8):2462-2469

3. Eom JM et al (2013) A comparative cross-sectional study on cosmetic outcomes after single port or conventional laparoscopic surgery. Eur J Obstet Gynecol Reprod Biol 167(1):104-109

4. Wang Tet al (2012) Comparison study of single-port (Octoport) and four-port total laparoscopic hysterectomy. Eur J Obstet Gynecol Reprod Biol 161(2):215-218

5. Choi YS et al (2013) Single-port vs. conventional multi-port access laparoscopy-assisted vaginal hysterectomy: comparison of surgical outcomes and complications. Eur J Obstet Gynecol Reprod Biol 169(2):366-369

6. Springborg H, Istre O (2012) Single port laparoscopic surgery: concept and controversies of a new technique. Acta Obstet Gynecol Scand 91(10): 1237-1240

7. Park HS et al (2011) Single-port access (SPA) laparoscopic surgery in gynecology: a surgeon's experience with an initial 200 cases. Eur J Obstet Gynecol Reprod Biol 154(1):81-84

8. Puntambekar S et al (2012) Single-incision total laparoscopic hysterectomy with conventional laparoscopy ports. Int J Gynaecol Obstet 117(1):37-39

9. Ichikawa $M$ et al (2011) Evaluation of laparoendoscopic single-site gynecologic surgery with a multitrocar access system. J Nippon Med Sch 78(4):235-240

10. Harmanli $\mathrm{OH}$ et al (2009) A comparison of short-term outcomes between laparoscopic supracervical and total hysterectomy. Am J Obstet Gynecol 201(5):536 e1-7

11. Mueller A et al (2009) Comparison of total laparoscopic hysterectomy (TLH) and laparoscopy-assisted supracervical hysterectomy (LASH) in women with uterine leiomyoma. Eur J Obstet Gynecol Reprod Biol 144(1):76-79

12. Cipullo L et al (2009) Laparoscopic supracervical hysterectomy compared to total hysterectomy. JSLS 13(3):370-375

13. Lieng $\mathrm{M}$ et al (2008) Long-term outcomes following laparoscopic supracervical hysterectomy. BJOG 115(13):1605-1610

14. Lieng $\mathrm{M}$ et al (2005) Outpatient laparoscopic supracervical hysterectomy with assistance of the lap loop. J Minim Invasive Gynecol 12(3):290-294

15. Kim TJ, Lee YY, An JJ, Choi CH, Lee JW, Kim BG, Bae DS (2012) Does single-port access (SPA) laparoscopy mean reduced pain? A retrospective cohort analysis between SPA and conventional laparoscopy. Elsevier Ireland Ltd, Ireland

16. Chen YJ, Wang PH, Ocampo EJ, Twu NF, Yen MS, Chao KC (2011) Single-port compared with conventional laparoscopic-assisted vaginal hysterectomy: a randomized controlled trial. Obstet Gynecol 117(4):906-912

17. Song $\mathrm{T}$ et al (2013) Cosmetic outcomes of laparoendoscopic singlesite hysterectomy compared with multi-port surgery: randomized controlled trial. J Minim Invasive Gynecol 20(4):460-467

18. Yim GW et al (2010) Transumbilical single-port access versus conventional total laparoscopic hysterectomy: surgical outcomes. Am J Obstet Gynecol 203(1):26 e1-6

19. Gunderson CC et al (2012) The risk of umbilical hernia and other complications with laparoendoscopic single-site surgery. J Minim Invasive Gynecol 19(1):40-45

20. Fader AN et al (2010) Laparoendoscopic single-site surgery in gynecology. Curr Opin Obstet Gynecol 22(4):331-338 